\title{
Can YouTube Video on Urological Problems Be Used As An Additional Resource in Telemedicine During the COVID-19 Pandemic?
}

\author{
(1) İsmail Selvi ${ }^{1}$, (1) Numan Baydilli2 \\ 1 University of Health Sciences Turkiye, Başakşehir Çam and Sakura City Hospital, Clinic of Urology, İstanbul, Turkiye \\ 2Erciyes University Faculty of Medicine, Department of Urology, Kayseri, Turkiye
}

What's known on the subject? and What does the study add?

Although establishing an international consensus about management of diagnosis and treatment of urological diseases during coronavirus disease-2019 (COVID-19) pandemic among urologists has been attempted, another important step is for patients to be informed about what they should do according to these algorithms-which symptoms are urgent versus cases where there is no need to consult a physician. Telemedicine method is not enough to reach and inform all urology patients. In this study, we aimed to evaluate whether the published YouTube videos are compatible with the current literature and whether they may be considered as a reliable additional information source for urological recommendations during COVID-19 period.

\begin{abstract}
Objective: Various recommendations are being offered in order of priority in the diagnosis and treatment of urological diseases during the coronavirus disease 2019 (COVID-19) period. Since it may not be possible to provide information to all patients through telemedicine, additional methods are needed to reach all patients. This study aimed to determine the reliability and quality of YouTube videos that provide information on urological disorders during the COVID-19 period. Another aim was to assess the most shared and most viewed videos on urological disorders on YouTube and their usability as an additional resource in telemedicine during the pandemic.

Materials and Methods: From 17 February 2020, to 31 July 2020, the following keywords were used to search videos on YouTube: "covid urology", "covid andrology", "covid fertility", "covid male infertility", "covid prostate", "covid bladder", "covid kidney", "covid renal", "covid testis", "covid cancer", "covid coitus", "covid sex", "covid sperm" and "covid erectile"; "coronavirus" was also used instead of "covid". In total, 232 videos were evaluated by two urologists according to video characteristics. The number of total views, views per day, likes, dislikes, comments, video length, date of video upload and duration on YouTube was recorded. Finally, a total of 136 relevant English videos were included in the study. A 5-point modified DISCERN tool was used to assess reliability, whereas a 5-point Global Quality score (GOS) was used to evaluate quality. Cohen's kappa score was used to determine inter-rater agreement, while the intraclass correlation coefficient was used to evaluate inter-observer reliability.

Results: All videos had a high DISCERN score [4, interquartile range (IOR) (3-5)] and GOS [5, IQR (4-5)] in general. Moreover, 20 (14.7\%) videos containing information about "sexual life" and "effects of COVID-19 on sperm" were described as debated. Videos uploaded by "universities/ professional organisations/non-profit physician/physician groups" had higher scores $(p<0.001)$. However, their rates of "number of views per day" $(p=0.036)$ and "likes" $(p<0.001)$ were lower. The majority of videos related to urology $(63.9 \%)$ included conversations about andrological disorders. The median number of total views and number of views per day were also highest for videos related to andrology.

Conclusion: Although andrological disorders are classified as a non-essential issue and andrological consultations are postponed during the COVID-19 period, the results show that the rate of YouTube videos related to andrology is even higher than uro-oncological diseases during this period. Therefore, it is important to share accurate and reliable information in this field. Our analysis shows that informative, easy-to-understand YouTube videos uploaded by "universities/professional organisations/non-profit physicians/physician groups" can be used as an additional method to telemedicine, especially for andrological disorders that do not require follow-up.
\end{abstract}

Keywords: COVID-19, SARS-CoV-2, telemedicine, urology, YouTube

Correspondence: İsmail Selvi MD, University of Health Sciences Turkiye, Başakşehir Çam and Sakura City Hospital, Clinic of Urology, İstanbul, Turkiye Phone: +90 2129096000 E-mail: ismselvi33@hotmail.com ORCID-ID: orcid.org/0000-0003-3578-0732

Received: 25.09 .2020

Accepted: 20.12 .2020

Cite this article as: Selvi İ, Baydilli N. Can YouTube Video on Urological Problems Be Used As An Additional Resource in Telemedicine During the CoVID-19 Pandemic? J Urol Surg 2021;7(2):69-85.

๑Copyright 2021 by the Association of Urological Surgery / Journal of Urological Surgery published by Galenos Publishing House. 


\section{Introduction}

Coronavirus disease-2019 (COVID-19), which is caused by severe acute respiratory syndrome-coronavirus-2, became evident following an increase in the number of pneumonia cases in China in December 2019 and later announced as a pandemic by the World Health Organization on 11 March 2020, as a result of its global rapid spread (1). All outpatient activities and surgeries except for emergency and oncological cases have been postponed or cancelled. Even oncological cases have been classified as low and high priority. Since the extent and duration of the pandemic are unpredictable, rescheduling is not possible during this period (2).

Urology, like all other fields, has been affected by this pandemic. Various strategies and recommendations are offered in a stepwise approach in order of priority for the diagnosis and treatment of urological diseases (2). Although establishing an international consensus among urologists has been attempted, another important step is to inform patients on what they should do according to these algorithms: which symptoms are urgent versus which cases do not require consultation to a physician. It is often possible to reach and inform patients with urological problems on follow-up by teleconsultation or videoconsultation system $(3,4)$. However, it is also important to inform people who are not on a urological follow-up programme about what they should do during the COVID-19 period when they encounter various urological symptoms.

During quarantine, the easiest and most practical source of information on any topic is the Internet. Since YouTube is an openaccess video-sharing website, it can disseminate information better than other social media tools (5). We have come across YouTube videos that highlight tasks that should be done during the COVID-19 period regarding urological diseases. Most of the videos are created by specialists in urology and by other healthcare providers. In this study, we aimed to evaluate which urological diseases people are curious about and want to learn during the COVID-19 period. By making a detailed analysis of YouTube videos related to what is needed for urological diseases during the COVID-19 period, we tried to identify the most shared and most viewed videos about urological pathologies on YouTube during the COVID-19 period. Another aim was to evaluate whether contents of YouTube videos are compatible with the current literature and whether they may be considered a reliable information source of urological recommendations during the COVID-19 period.

\section{Materials and Methods}

\section{Data Collection}

The following keywords were used to search on YouTube (http://www.youtube.com): "covid urology", "covid andrology", "covid fertility", "covid male infertility", "covid prostate", "covid bladder", "covid kidney", "covid renal", "covid testis", "covid cancer", "covid coitus", "covid sex", "covid sperm" and "covid erectile". The same search was performed using the term "coronavirus" instead of "covid". The videos were sorted according to relevance using a YouTube filtering system. We found 232 videos related to "covid" and "urological conditions" between 17 February 2020 and 31 July 2020, and evaluated them for suitability for our study. All videos were saved in a playlist for review on 31 July 2020, since search results can vary every day on YouTube. Two independent urologists with board certification (Fellow of the European Board of Urology) (I.S. and N.B.) viewed and analysed the videos. Both urologists were blinded to each other's scores on video assessments.

\section{Inclusion Criteria for Videos}

The main subjects of our study are lay people who are not familiar with medical concepts and terms. These people need videos that provide simple, basic information in an easily understandable language on what they should do when they experience urological symptoms or disease during the COVID-19 period. For this purpose, English videos having accurate, comprehensive and easily understandable information for lay people rather than containing medical terms and scientific data were included in the study.

\section{Exclusion Criteria for Videos}

In total, 61 videos mentioned recommendations of the European Urology Association (EAU) guidelines and American Urological Association (AUA) guidelines for urologists using medical concepts and scientific terms. Since they were not suitable for the study purpose, we described them as "irrelevant", and they were excluded from the study. Non-English videos and videos without audio or visuals were also removed from the study. These data collection criteria are similar to previous studies evaluating YouTube videos on different topics $(6,7)$.

\section{Evaluation of Video Characteristics}

For each YouTube video, the number of total views, views per day, likes, dislikes, comments, video length, date of video upload and duration on YouTube were recorded. The accuracy of the information in the videos was evaluated according to the EAU and AUA guidelines (8-10). When evaluating videos on infertility, the American Society for Reproductive Medicine guidelines and the European Society of Human Reproduction and Embryology guidelines were considered, in addition to the two aforementioned guidelines $(11,12)$.

Videos containing scientific, accurate and comprehensive information on the diagnosis and treatment of urological diseases during the COVID-19 period that have been agreed upon according to the aforementioned guidelines were 
defined as useful information. Since there are some topics with controversial ideas about diagnosis and treatment that have not been agreed on, videos that did not fully convey controversial or uncertain issues and did not make a balanced and neutral assessment were defined as debated information (e.g. How to lead a reliable sexual life during the COVID-19 period?) Many videos mentioned guidelines of the "Centers for Disease Control and Prevention" to inform about whether COVID-19 causes sexually transmitted disease after intercourse (13), but some videos did not. COVID-19 does not cause sexually transmitted disease, but physical contact during intercourse may cause COVID-19 transmission. Therefore, having sex with your spouse or the same partner who is asymptomatic is safer than having sex with a foreign partner. However, no current evidence-based guidelines are available. The classification made according to the source of the video was as follows: universities/ professional organisations/non-profit physicians/physicians (source 1), stand-alone health information websites (source 2), medical advertisements/for-profit organisations (source 3) and individual users (source 4). Other classifications were made according to the target audience (female/male/both genders) and the speaker in the video (physician/non-physician health provider/individual in the video/external voice).

\section{Scoring System for Video Evaluation}

The original DISCERN tool is a validated tool comprising 16 questions. It evaluates the quality of written health information regarding treatment options (14). It includes questions evaluating the reliability of written documents, such as information leaflets, discussing treatment options. The main items of this tool are useful to evaluate the aims and clarity of written information sources and whether the written documents are sufficient to access support for shared decision-making (15).

Since the DISCERN tool was created long before online YouTube videos became popular, one of the most recent publications stated that the items of the original DISCERN tool are insufficient to evaluate videos (15). By contrast, many studies on identifying educationally useful YouTube videos have pointed out that the modified DISCERN tool and Global Quality score (GOS) provide a more suitable assessment of visual, vocal and verbal information, scientific accuracy of content and clarity of the given massage $(6,7,16)$.

A 5-point modified DISCERN tool was used to evaluate the video reliability and quality of health information available to patients as in previous studies on YouTube videos (Table 1) $(6,7)$ GOS was also used in our study. This 5-point scale is used to evaluate the accessibility, quality and overall flow of information within videos from website resources. A score of "1" indicates "poor quality", and a score of " 5 " indicates "excellent quality" (Table 1) $(6,7)$. After each urologist evaluated and scored the videos, Cohen's kappa score was used to determine inter-rater agreement, while the intra-class correlation coefficient was used to evaluate inter-observer reliability.

\section{Statistical Analysis}

During the analysis of video characteristics and categorisation of video contents, each data related to videos were assessed as independent variables. The normality of these variables was evaluated using Shapiro-Wilk test, Kolmogorov-Smirnov test, histogram and 0-0 plots. Levene's test was used to assess variance homogeneity. Variables not showing normal distribution are expressed as median $\left(25^{\text {th }}-75^{\text {th }}\right.$ percentile). Categorical variables are shown as number and per cent $(n, \%)$. The Mann-Whitney U test was used to analyse variables with non-normal distribution between the two groups. All analyses were made using IBM SPSS Statistics 22 (IBM Corp., Armonk, NY, USA) software package; $p<0.05$ was considered significant.

\section{Results}

\section{General Information on Video Characteristics}

After evaluating a total of 232 videos related to "COVID" and various "urological diseases" using the search keywords on YouTube, 136 videos were selected according to the inclusion criteria. The flowchart of the study design is shown in Figure 1. The median duration from the date of upload in YouTube, between 17 February 2020 and 31 July 2020, was 47 (min-max, 3-166) days.

\section{Evaluation of Videos According to the Inter-rater Agreement and Intra-class Correlation}

The level of inter-rater agreement in terms of the usefulness assessment of videos was positive (kappa coefficient $=0.903$ ). The intra-class correlation coefficient was calculated as 0.941 (95\% confidence interval 0.834-0.989) for the DISCERN reliability score and 0.961 (95\% confidence interval 0.890-0.991) for GOS. The results show a high inter-rater reliability.

\section{Classification of YouTube Videos According to Their Reliability}

Controversial ideas still exist on sexual life during the COVID-19 period. A total of $20(14.7 \%)$ videos that did not talk about contradicting comments on topics described in a balanced and neutral way were evaluated as "debated". Of these videos, four focused on the effects of COVID-19 on sperm and 16 were about sexual intercourse during the COVID-19 period. Content of videos related to all other topics was evaluated as "useful information".

\section{Assessment of Video Characteristics, Sources of Video Upload and Speakers}

Table 2 shows the characteristics of the videos evaluated. Although all videos had high DISCERN score and GOS in general, 


\section{Enrollment}

\section{Allocation}

Follow-up and Analysis
Keywords of "covid urology", "covid andrology", "covid fertility", "covid male infertility", "covid prostate", "covid bladder", "covid kidney", "covid renal", "covid testis", "covid cancer", "covid coitus", "covid sex", "covid sperm", "covid erectile" and also "coronavirus" as well as "covid" were searched and all related videos were reviewed

\section{Excluded videos $(n=96)$}

- Irrelevant $(n=61)$

- Non-English $(\mathrm{n}=17)$

- No audio $(\mathrm{n}=\mathbf{1 0})$

- $\operatorname{No}$ visual $(\mathrm{n}=8)$
Eligible videos included

$(n=136)$
Videos were defined according to the usefulness of information

- Useful information $(n=116,85.3 \%)$

- Debated information ( $\mathrm{n}=\mathbf{2 0}, \mathbf{1 4 . 7} \%)$

\section{Videos were categorized in terms of sources of the uploads}

- Universities/professional organizations/nonprofit\# Physicians/physicians ( $\mathrm{n}=\mathbf{1 0 5}, \mathbf{7 7 . 2} \%)$

- Medicaladvertisements/for-profit-organizations ( $n=1,0.7 \%)$

- Individualusers/patients ( $\mathrm{n}=\mathbf{3 0 , 2 2 . 1 \% )}$

Figure 1. Flowchart of the study design

Videos were categorized according to video content

- Andrology $(n=87,63.9 \%)$

- Uro-oncology $(n=26,19.1 \%)$

- Renaltransplantation $(n=8,5.9 \%)$

- General urology/other topics $(n=15,11.1 \%)$ 
Table 1. Evaluation tools for reliability and quality of YouTube videos that give information on urological disorders during the COVID-19 period

\section{DISCERN Reliability Tool ( 1 point per question if answered yes)}

1. Are the explanations given in the video clear and understandable?

2. Are useful reference sources given? (publication cited, from valid studies)

3. Is the information in the video balanced and neutral?

4. Are additional sources of information given from which the viewer can benefit?

5. Does the video evaluate areas that are controversial or uncertain?

\section{Global Quality scale}

1. Poor quality, poor flow, most information missing and not helpful for patients

2. Generally poor and some information given but of limited use to patients

3. Moderate quality and some important information is adequately discussed

4. Good quality, good flow, most relevant information is covered and useful for patients

5. Excellent quality, excellent flow and very useful for patients COVID-19: Coronavirus disease-2019

videos uploaded by individual users and medical advertisement/ for-profit companies (22.8\%) had lower scores. However, the rates of "the number of views per day" and "likes" were higher in the latter group (Table 3). Most of the sources of videos were universities/professional organisations/non-profit physician/ physician groups $(77.2 \%)$, while the physicians and nonphysician health providers $(83.8 \%)$ made up the majority of the speakers (Table 2). Table 4 summarises the characteristics of YouTube videos.

\section{Assessment of Video Contents Related to Different Urological Disorders}

When the video content was categorised as andrology, urooncology, renal transplantation and general urology/other topics, videos were distributed as $63.9 \%, 19.1 \%, 5.9 \%$ and $11.1 \%$, respectively. The median number of total views and numbers of views per day were highest for videos related to andrology. The lowest numbers were observed for videos related to uro-oncology (Figure 2).

\section{Discussion}

All outpatient activities, except emergency cases, have been suspended and surgical activities, except for emergency and/ or oncological interventions, have been limited. How long this unexpected pandemic will continue and its socioeconomic and psychological burden will be alleviated is unknown. In some
Table 2. Analysis of video characteristics according to their usefulness

\begin{tabular}{|c|c|}
\hline Parameters & $n=136$ \\
\hline Number of total views & $286.50(82-1457.50)$ \\
\hline Video length (second) & $296.50(141.50-923)$ \\
\hline Duration on YouTube (day) & $47(32-57.75)$ \\
\hline Number of views per day & $8.20(2.14-31.56)$ \\
\hline Likes & $5(1-25)$ \\
\hline Dislikes & $0(0-1)$ \\
\hline Comments & $1(0-5)$ \\
\hline DISCERN score & $4(3-5)$ \\
\hline Global Quality Score & $5(4-5)$ \\
\hline \multicolumn{2}{|l|}{ Video content, $n(\%)$} \\
\hline -Infertility & $57(41.9)$ \\
\hline -Sexual life & $26(19.1)$ \\
\hline -Prostate cancer & $15(11.1)$ \\
\hline $\begin{array}{l}\text {-Telemedicine in urology and general } \\
\text { urology }\end{array}$ & $10(7.4)$ \\
\hline -Bladder cancer & $8(5.9)$ \\
\hline -Renal transplantation & $8(5.9)$ \\
\hline -Effects of COVID-19 on sperm & $4(2.9)$ \\
\hline -Renal cancer & $3(2.2)$ \\
\hline -Effects of COVID-19 on testis & $2(1.5)$ \\
\hline -Neurogenic bladder & $1(0.7)$ \\
\hline -Benign renal diseases & $1(0.7)$ \\
\hline -Effects of COVID-19 on kidney physiology & $1(0.7)$ \\
\hline \multicolumn{2}{|l|}{ Sources of uploads, n (\%) } \\
\hline $\begin{array}{l}\text {-Universities/professional organisations/ } \\
\text { non-profit physician/physician groups }\end{array}$ & $105(77.2)$ \\
\hline -Stand-alone health information websites & $0(0.0)$ \\
\hline $\begin{array}{l}\text {-Medical advertisement/for-profit } \\
\text { companies }\end{array}$ & $1(0.7)$ \\
\hline -Individual users & $30(22.1)$ \\
\hline \multicolumn{2}{|l|}{ Speakers, n (\%) } \\
\hline -Physician & $102(75.0)$ \\
\hline -Non-physician healthcare provider & $12(8.8)$ \\
\hline -Individual in the video & $15(11.1)$ \\
\hline -External voice & $7(5.1)$ \\
\hline \multicolumn{2}{|l|}{ Target audience, $\mathrm{n}(\%)$} \\
\hline -Male & $5(3.7)$ \\
\hline -Both male and female & $131(96.3)$ \\
\hline \multicolumn{2}{|l|}{ Usefulness of source, n (\%) } \\
\hline - Useful information & $116(85.3)$ \\
\hline - Debated information & $20(14.7)$ \\
\hline \multicolumn{2}{|c|}{$\begin{array}{l}\text { Non-normally distributed data are expressed as "median }\left(25^{\text {th }}-75^{\text {th }} \text { percentile)" }\right. \\
\text { Categorical data are expressed as "number (percent)", COVID-19: Coronavirus disease } \\
-2019\end{array}$} \\
\hline
\end{tabular}


countries, teams of national experts discuss and try to reorganise possible strategies for patients with urological disorders $(3,17)$.

This pandemic has made "telemedicine" more prominent as a pragmatic approach to reduce the risk of disease transmission. A virtual clinic is formed between physicians and patients so that

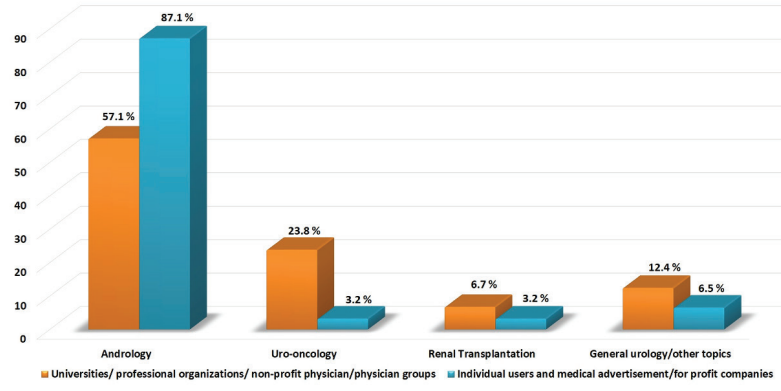

Figure 2. Distribution of video contents according to sources of uploads problems not needing a traditional face-to-face consultation may be evaluated while avoiding a hospital visit $(17,18)$. Various electronic communication devices such as telephone calls, video conferences and online email consultations can be developed for telemedicine (19-21). In this way, telemedicine, which is a very pragmatic option, especially for infectious diseases and public health emergencies, is also on the agenda for urology practice (19).

During this period, Luciani et al. (19) observed that about half of the patients had to cancel their doctor's appointment. The rate of face-to-face visits dramatically declined from 63\% to $9 \%$ within the first 4 weeks of the pandemic.

Moreover, the majority (94.5\%) of patients with urology diseases are at risk for a severe course of COVID-19 because of their advanced age and comorbidities, so social isolation is

Table 3. Analysis of video characteristics according to sources of uploads

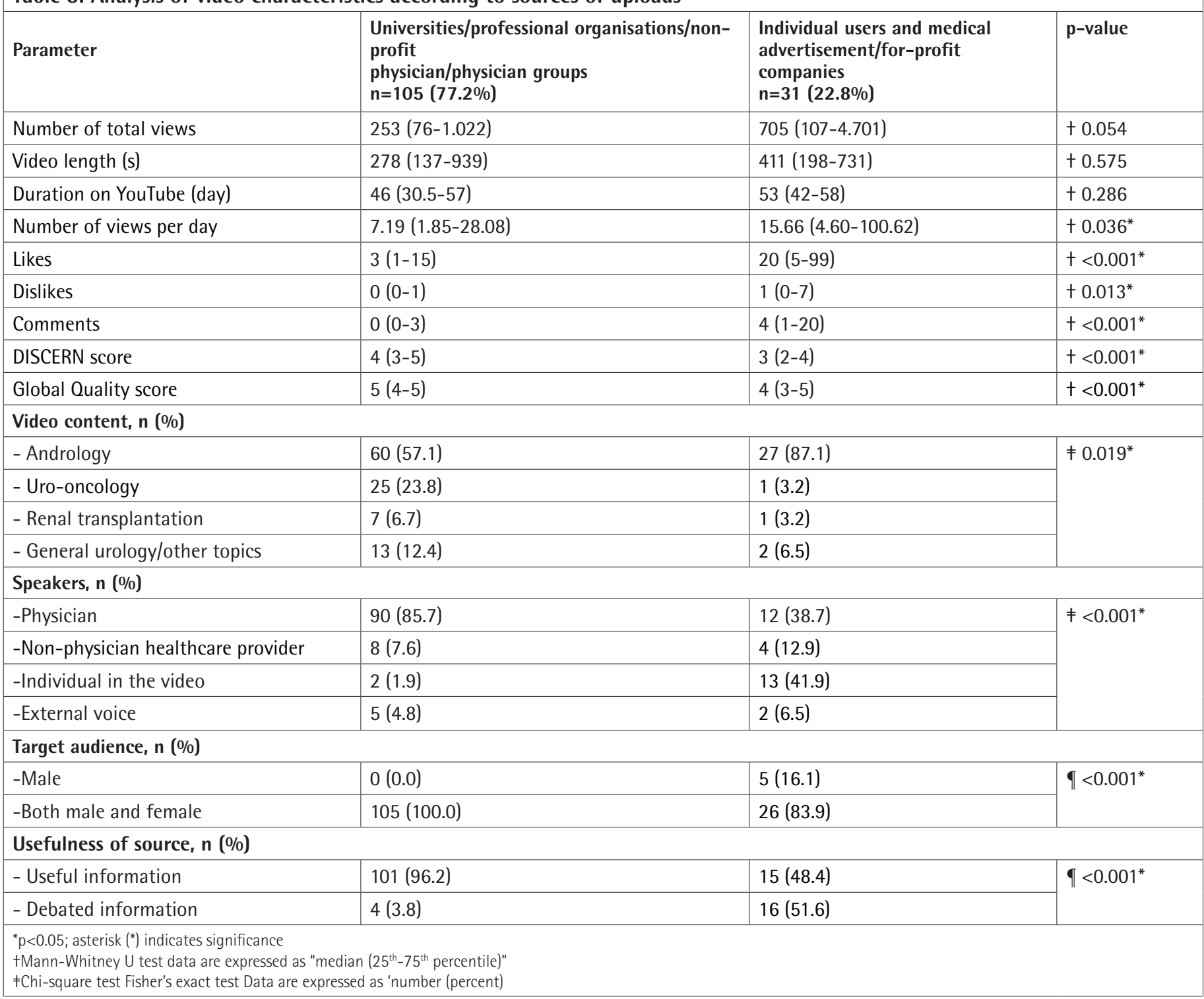




\begin{tabular}{|c|c|c|c|c|c|c|c|c|c|c|c|c|}
\hline 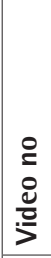 & 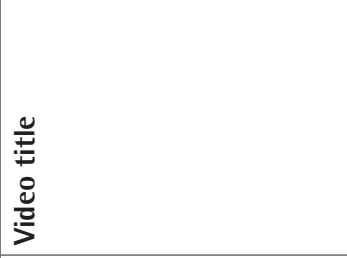 & $\overrightarrow{\check{g}}$ & 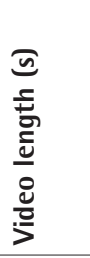 & 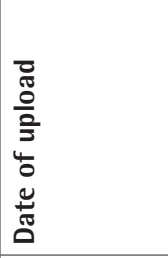 & 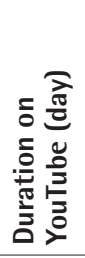 & 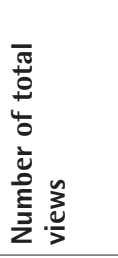 & 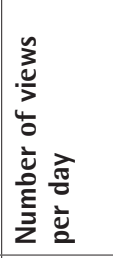 & 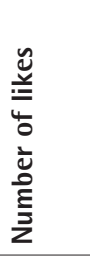 & 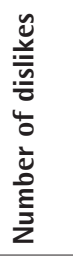 & 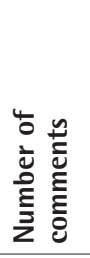 & 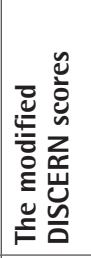 & 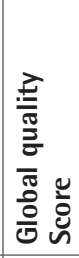 \\
\hline 1 & $\begin{array}{l}\text { How to have safe sex } \\
\text { during the quarantine }\end{array}$ & $\begin{array}{l}\text { https://youtu.be/ } \\
\text { sBXZCZy3E-0 }\end{array}$ & 320 & 24.03 .2020 & 55 & 341809 & 6214.7 & 8200 & 270 & 656 & 3 & 2 \\
\hline 2 & $\begin{array}{l}\text { Love in the time of } \\
\text { coronavirus (washing your } \\
\text { hands is sexy now!) }\end{array}$ & $\begin{array}{l}\text { https://youtu. } \\
\text { be/6_tsEVOf7ro }\end{array}$ & 133 & 12.03 .2020 & 67 & 262425 & 3916.79 & 2800 & 98 & 118 & 1 & 1 \\
\hline 3 & $\begin{array}{l}\text { Stockpiling semen to } \\
\text { fight the coronavirus }\end{array}$ & $\begin{array}{l}\text { https://youtu.be/ } \\
\text { Q7R01ZYvCtg }\end{array}$ & 298 & 13.03 .2020 & 66 & 68453 & 1037.16 & 273 & 135 & 173 & 1 & 1 \\
\hline 4 & $\begin{array}{l}\text { Sex during } \\
\text { coronavirus?|NBCLX }\end{array}$ & $\begin{array}{l}\text { https://youtu.be/ } \\
\text { cDA9ty2aAD0 }\end{array}$ & 244 & 27.03.2020 & 52 & 66222 & 1273.5 & 152 & 21 & 40 & 4 & 4 \\
\hline 5 & $\begin{array}{l}\text { Dr. Oz says regular sex } \\
\text { during coronavirus } \\
\text { quarantine is good|TMZ } \\
\text { TV }\end{array}$ & $\begin{array}{l}\text { https://youtu.be/ } \\
\text { CIEWmyZFtyk }\end{array}$ & 106 & 18.03.2020 & 61 & 59218 & 970.78 & 715 & 48 & 167 & 2 & 3 \\
\hline 6 & $\begin{array}{l}\text { Trying to get pregnant } \\
\text { during coronavirus [TTC } \\
\text { MUST WATCH] }\end{array}$ & $\begin{array}{l}\text { https://youtu.be/ } \\
\text { sZvZK3Bn1zA }\end{array}$ & 1380 & 17.03 .2020 & 62 & 37054 & 597.64 & 533 & 13 & 168 & 5 & 5 \\
\hline 7 & & $\begin{array}{l}\text { This video is no } \\
\text { longer available } \\
\text { because the } \\
\text { YouTube account } \\
\text { associated with } \\
\text { this video has } \\
\text { been terminated }\end{array}$ & 133 & 17.03 .2020 & 62 & 31588 & 509.48 & 471 & 22 & 125 & 2 & 3 \\
\hline 8 & $\begin{array}{l}\text { Coronavirus means } \\
\text { some couples may never } \\
\text { conceive as COVID-19 } \\
\text { puts IVF on hold|ITV News }\end{array}$ & $\begin{array}{l}\text { https://youtu.be/ } \\
\text { E7KialUwlbE }\end{array}$ & 195 & 28.03.2020 & 51 & 26071 & 511.19 & 126 & 74 & 0 & 3 & 5 \\
\hline 9 & $\begin{array}{l}\text { Coronavirus outbreak: } \\
\text { Dating and sex in the } \\
\text { time of a pandemic - } \\
\text { Wait there's more podcast }\end{array}$ & $\begin{array}{l}\text { https://youtu. } \\
\text { be/3VRV3hqsUCM }\end{array}$ & 1420 & 24.03.2020 & 55 & 19416 & 353.01 & 149 & 58 & 92 & 3 & 4 \\
\hline 10 & $\begin{array}{l}\text { Dr. Oz talks possible } \\
\text { treatments and drugs for } \\
\text { coronavirus, sex during } \\
\text { quarantine and more }\end{array}$ & $\begin{array}{l}\text { https://youtu.be/ } \\
\text { Bz_QEeeAvlw }\end{array}$ & 246 & 19.03.2020 & 60 & 17786 & 296.43 & 159 & 13 & 26 & 3 & 4 \\
\hline 11 & $\begin{array}{l}\text { Couples having } \\
\text { sex [DURING THE } \\
\text { QUARANTINE SEXY } \\
\text { TIMES]! }\end{array}$ & $\begin{array}{l}\text { https://youtu. } \\
\text { be/_eA8sr_NdOo }\end{array}$ & 450 & 26.03.2020 & 53 & 13155 & 248.2 & 40 & 7 & 1 & 3 & 4 \\
\hline 12 & & $\begin{array}{l}\text { This video is no } \\
\text { longer available } \\
\text { because the } \\
\text { YouTube account } \\
\text { associated with } \\
\text { this video has } \\
\text { been terminated }\end{array}$ & 274 & 19.03 .2020 & 60 & 12913 & 215.21 & 49 & 4 & 8 & 3 & 4 \\
\hline 13 & $\begin{array}{l}\text { Your coronavirus } \\
\text { questions answered: What } \\
\text { about IVF treatments and } \\
\text { chemotherapy?|TODAY }\end{array}$ & $\begin{array}{l}\text { https://youtu.be/ } \\
\text { VsTr6zroLsl }\end{array}$ & 295 & 19.03 .2020 & 60 & 12790 & 213.16 & 78 & 5 & 21 & 5 & 5 \\
\hline 14 & $\begin{array}{l}\text { Is coronavirus sexually } \\
\text { transmitted? Experts } \\
\text { respond }\end{array}$ & $\begin{array}{l}\text { https://youtu.be/ } \\
\text { Fzwdu-D5BOw }\end{array}$ & 558 & 22.03 .2020 & 57 & 12277 & 215.38 & 40 & 1 & 7 & 4 & 5 \\
\hline
\end{tabular}




\begin{tabular}{|c|c|c|c|c|c|c|c|c|c|c|c|c|}
\hline 15 & $\begin{array}{l}\text { COVID-19: What } \\
\text { transplant patients need } \\
\text { to know }\end{array}$ & $\begin{array}{l}\text { https://youtu.be/ } \\
\text { IXMbKt1ZcT4 }\end{array}$ & 272 & 13.03 .2020 & 66 & 9950 & 150.75 & 92 & 6 & 0 & 4 & 5 \\
\hline 16 & $\begin{array}{l}\text { COVID-19 and kidney } \\
\text { disease }\end{array}$ & $\begin{array}{l}\text { https://youtu.be/ } \\
\text { ELAPITCMF30 }\end{array}$ & 191 & 12.03 .2020 & 67 & 7369 & 109.98 & 61 & 10 & 9 & 3 & 4 \\
\hline 17 & $\begin{array}{l}\text { COVID } 19 \text {, what to ask } \\
\text { your fertility doctor }\end{array}$ & $\begin{array}{l}\text { https://youtu.be/ } \\
\text { RuaSYpUPt-0 }\end{array}$ & 1175 & 25.03 .2020 & 54 & 6058 & 112.18 & 152 & 11 & 20 & 4 & 5 \\
\hline 18 & $\begin{array}{l}\text { Dr. Oz suggests couples } \\
\text { 'have sex' amidst } \\
\text { coronavirus chaos }\end{array}$ & $\begin{array}{l}\text { https://youtu.be/ } \\
\text { ht9LqsB_Nmc }\end{array}$ & 83 & 20.03 .2020 & 59 & 5937 & 100.62 & 99 & 8 & 20 & 2 & 3 \\
\hline 19 & $\begin{array}{l}\text { NY Department of health } \\
\text { releases COVID-19 sex } \\
\text { guide }\end{array}$ & $\begin{array}{l}\text { https://youtu.be/ } \\
\text { Y35pukvFNJo }\end{array}$ & 152 & 26.03 .2020 & 53 & 5519 & 104.13 & 30 & 3 & 7 & 5 & 5 \\
\hline 20 & $\begin{array}{l}\text { Sex cannot transmit virus, } \\
\text { but one can be infected } \\
\text { because of proximity - } \\
\text { Masika }\end{array}$ & $\begin{array}{l}\text { https://youtu.be/ } \\
\text { KeS6iMGQfFA }\end{array}$ & 1140 & 20.03.2020 & 59 & 5410 & 91.69 & 17 & 1 & 3 & 3 & 4 \\
\hline 21 & & $\begin{array}{l}\text { This video is no } \\
\text { longer available } \\
\text { because the } \\
\text { YouTube account } \\
\text { associated with } \\
\text { this video has } \\
\text { been terminated }\end{array}$ & 475 & 26.03.2020 & 53 & 4701 & 88.69 & 382 & 3 & 89 & 1 & 2 \\
\hline 22 & $\begin{array}{l}\text { Coronavirus and getting } \\
\text { pregnant|A fertility } \\
\text { doctor talks about } \\
\text { COVID-19 }\end{array}$ & $\begin{array}{l}\text { https://youtu.be/ } \\
\text { D8jsKnyH000 }\end{array}$ & 731 & 13.03 .2020 & 66 & 3482 & 52.75 & 36 & 1 & 13 & 5 & 5 \\
\hline 23 & & $\begin{array}{l}\text { This video is no } \\
\text { longer available } \\
\text { because the } \\
\text { YouTube account } \\
\text { associated with } \\
\text { this video has } \\
\text { been terminated }\end{array}$ & 123 & 17.02 .2020 & 90 & 3375 & 37.5 & 39 & 4 & 28 & 3 & 4 \\
\hline 24 & $\begin{array}{l}\text { Practical advice FOR } \\
\text { prostate cancer PATIENTS } \\
\text { for weathering changes } \\
\text { caused by COVID-19 }\end{array}$ & $\begin{array}{l}\text { https://youtu.be/ } \\
\text { v4JyRwNYVZU }\end{array}$ & 1104 & 27.03 .2020 & 52 & 2937 & 56.48 & 15 & 0 & 1 & 5 & 5 \\
\hline 25 & $\begin{array}{l}\text { IVF decisions in the } \\
\text { time of COVID-19 } \\
\text { (Coronavirus): Advice for } \\
\text { fertility patients }\end{array}$ & $\begin{array}{l}\text { https://youtu. } \\
\text { be/1nD_SVpHHs8 }\end{array}$ & 590 & 23.03 .2020 & 56 & 2493 & 44.51 & 42 & 2 & 3 & 4 & 5 \\
\hline 26 & $\begin{array}{l}\text { Tips on how to not } \\
\text { destroy your relationship } \\
\text { while in quarantine for } \\
\text { coronavirus|New York } \\
\text { Post }\end{array}$ & $\begin{array}{l}\text { https://youtu.be/ } \\
\text { ysDdWVu6c5A }\end{array}$ & 120 & 27.03.2020 & 52 & 2329 & 44.78 & 38 & 3 & 10 & 3 & 4 \\
\hline 27 & $\begin{array}{l}\text { Male infertility \&t } \\
\text { COVID-19 Infection, what } \\
\text { do we know! }\end{array}$ & $\begin{array}{l}\text { https://youtu.be/ } \\
\text { w9u-N8eGU0k }\end{array}$ & 2545 & 8.04 .2020 & 44 & 2185 & 49.65 & 110 & 2 & 3 & 5 & 5 \\
\hline 28 & $\begin{array}{l}\text { Coronavirus: Men } \\
\text { infected with COVID-19 } \\
\text { may experience testicular } \\
\text { damage }\end{array}$ & $\begin{array}{l}\text { https://youtu.be/ } \\
\text { cun9TJNiOCc }\end{array}$ & 105 & 14.03 .2020 & 65 & 2073 & 31.89 & 19 & 5 & 25 & 4 & 4 \\
\hline 29 & $\begin{array}{l}\text { COVID-19: Dr. Michael } \\
\text { Braun discusses the virus' } \\
\text { impact on patients with } \\
\text { renal (kidney) disease }\end{array}$ & $\begin{array}{l}\text { https://youtu.be/ } \\
\text { j4V950M87Jw }\end{array}$ & 155 & 13.03 .2020 & 66 & 1955 & 29.62 & 12 & 0 & 3 & 3 & 4 \\
\hline
\end{tabular}




\begin{tabular}{|c|c|c|c|c|c|c|c|c|c|c|c|c|}
\hline 30 & $\begin{array}{l}\text { Sex, sexuality and } \\
\text { COVID-19. An open } \\
\text { conversation }\end{array}$ & $\begin{array}{l}\text { https://youtu.be/ } \\
\text { ShH7qn3wx8Y }\end{array}$ & 459 & 19.03 .2020 & 58 & 1674 & 28.86 & 20 & 0 & 3 & 3 & 3 \\
\hline 31 & $\begin{array}{l}\text { Telehealth during } \\
\text { COVID-19 }\end{array}$ & $\begin{array}{l}\text { https://youtu.be/ } \\
\text { Kx09-tuXUuw }\end{array}$ & 3603 & 30.03 .2020 & 49 & 1645 & 33.57 & 15 & 1 & 6 & 5 & 5 \\
\hline 32 & $\begin{array}{l}\text { COVID-19 and the } \\
\text { kidney, why are we } \\
\text { seeing so much kidney } \\
\text { involvement? }\end{array}$ & $\begin{array}{l}\text { https://youtu.be/ } \\
\text { capCRENmDMc }\end{array}$ & 654 & 26.03 .2020 & 53 & 1621 & 30.58 & 42 & 1 & 35 & 3 & 4 \\
\hline 33 & $\begin{array}{l}\text { Coronavirus \&t fertility } \\
\text { expert Q\&A }\end{array}$ & $\begin{array}{l}\text { https://youtu.be/ } \\
\text { ilv_mGDo7xo }\end{array}$ & 509 & 24.03 .2020 & 55 & 1574 & 28.61 & 19 & 2 & 6 & 5 & 5 \\
\hline 34 & $\begin{array}{l}\text { Coronavirus causes male } \\
\text { infertility: What they are } \\
\text { not telling you }\end{array}$ & $\begin{array}{l}\text { https://youtu.be/ } \\
\text { NjcO_pNedXE }\end{array}$ & 171 & 29.02 .2020 & 78 & 1477 & 18.93 & 25 & 0 & 6 & 4 & 4 \\
\hline 35 & $\begin{array}{l}\text { What prostate cancer } \\
\text { patients need to know } \\
\text { about treatment during } \\
\text { the COVID-19 pandemic }\end{array}$ & $\begin{array}{l}\text { https://youtu.be/ } \\
\text { UkCfLFotpag }\end{array}$ & 779 & 16.04 .2020 & 32 & 1399 & 43.71 & 3 & 0 & 1 & 5 & 5 \\
\hline 36 & $\begin{array}{l}\text { IVF treatments put on } \\
\text { hold amid coronavirus } \\
\text { concerns }\end{array}$ & $\begin{array}{l}\text { https://youtu.be/ } \\
\text { ujF9EuGWUwo }\end{array}$ & 96 & 20.03 .2020 & 59 & 1328 & 22.5 & 8 & 3 & 4 & 5 & 5 \\
\hline 37 & $\begin{array}{l}\text { COVID-19 - Is it safe } \\
\text { to conceive during the } \\
\text { outbreak? }\end{array}$ & $\begin{array}{l}\text { https://youtu.be/ } \\
\text { Z3W03HNE-go }\end{array}$ & 268 & 4.04 .2020 & 44 & 1318 & 29.95 & 18 & 0 & 2 & 4 & 5 \\
\hline 38 & $\begin{array}{l}\text { What fertility patients } \\
\text { should know about } \\
\text { COVID-19 (Coronavirus) }\end{array}$ & $\begin{array}{l}\text { https://youtu.be/ } \\
\text { svpKGG1Mfjk }\end{array}$ & 356 & 23.03 .2020 & 56 & 1299 & 23.19 & 17 & 2 & 6 & 4 & 5 \\
\hline 39 & $\begin{array}{l}\text { How to continue your } \\
\text { fertility journey during } \\
\text { the COVID- } 19 \text { crisis: } 3 \\
\text { pieces of advice }\end{array}$ & $\begin{array}{l}\text { https://youtu.be/ } \\
\text { raKGuR0003Y }\end{array}$ & 607 & 1.04 .2020 & 47 & 1179 & 25.08 & 32 & 0 & 5 & 4 & 5 \\
\hline 40 & COVID-19 \&t your fertility & $\begin{array}{l}\text { https://youtu.be/ } \\
\text { zqdZssWwkMg }\end{array}$ & 1114 & 26.03 .2020 & 54 & 865 & 16.01 & 0 & 0 & 0 & 5 & 5 \\
\hline 41 & $\begin{array}{l}\text { How COVID-19 is } \\
\text { affecting transplants }\end{array}$ & $\begin{array}{l}\text { https://youtu.be/ } \\
\text { y0VEC6hVbkl }\end{array}$ & 119 & 30.03 .2020 & 49 & 653 & 13.32 & 12 & 0 & 2 & 5 & 5 \\
\hline 42 & $\begin{array}{l}\text { ZERO update: COVID-19 \&t } \\
\text { prostate cancer }\end{array}$ & $\begin{array}{l}\text { https://youtu.be/ } \\
\text { RHkkNaxj2zY }\end{array}$ & 167 & 13.03 .2020 & 66 & 846 & 12.81 & 5 & 1 & 0 & 4 & 4 \\
\hline 43 & $\begin{array}{l}\text { COVID-19: Impact on } \\
\text { kidney disease and dialysis }\end{array}$ & $\begin{array}{l}\text { https://youtu.be/ } \\
\text { ijqd4sol_IU }\end{array}$ & 242 & 23.03.2020 & 56 & 439 & 7.83 & 11 & 0 & 0 & 4 & 5 \\
\hline 44 & $\begin{array}{l}\text { COVID-19, fertility and } \\
\text { pregnancy: Coronavirus } \\
\text { updates from a fertility } \\
\text { doctor }\end{array}$ & $\begin{array}{l}\text { https://youtu.be/ } \\
\text { Q0E4qScEOSk }\end{array}$ & 948 & 1.04 .2020 & 47 & 795 & 16.91 & 17 & 0 & 6 & 5 & 5 \\
\hline 45 & $\begin{array}{l}\text { COVID-19|A urologist's } \\
\text { perspective and plan }\end{array}$ & $\begin{array}{l}\text { https://youtu.be/ } \\
\text { OfveW-OH7LY }\end{array}$ & 374 & 19.03 .2020 & 60 & 764 & 12.73 & 25 & 1 & 2 & 4 & 5 \\
\hline 46 & $\begin{array}{l}\text { The responsibilities of } \\
\text { an IVF centre during the } \\
\text { COVID-19 pandemic: } \\
\text { CHR explains the ASRM } \\
\text { statement }\end{array}$ & $\begin{array}{l}\text { https://youtu.be/ } \\
\text { j5rV8f_aLHg }\end{array}$ & 707 & 1.04 .2020 & 47 & 738 & 15.7 & 9 & 1 & 1 & 5 & 5 \\
\hline 47 & $\begin{array}{l}\text { Coronavirus \& fertility: } \\
\text { fact vs fiction with Dr. } \\
\text { Cindy Duke }\end{array}$ & $\begin{array}{l}\text { https://youtu.be/ } \\
\text { oXoaPA9NmcY }\end{array}$ & 2407 & 23.04 .2020 & 25 & 732 & 29.28 & 27 & 0 & 0 & 5 & 5 \\
\hline 48 & $\begin{array}{l}\text { Is having sex during } \\
\text { COVID-19 outbreak safe? } \\
\text { Know from the experts }\end{array}$ & $\begin{array}{l}\text { https://youtu.be/ } \\
\text { XRY7-aHWkWc }\end{array}$ & 138 & 4.04 .2020 & 44 & 677 & 15.38 & 5 & 0 & 0 & 2 & 3 \\
\hline 49 & $\begin{array}{l}\text { COVID-19 CANCELLED } \\
\text { OUR IVF FROZEN } \\
\text { TRANSFER // Coping with } \\
\text { a cancelled IVF cycle due } \\
\text { to Corona Virus }\end{array}$ & $\begin{array}{l}\text { https://youtu.be/ } \\
\text { LPSmBF02DDg }\end{array}$ & 768 & 3.04 .2020 & 45 & 705 & 15.66 & 26 & 3 & 12 & 3 & 4 \\
\hline
\end{tabular}




\begin{tabular}{|c|c|c|c|c|c|c|c|c|c|c|c|c|}
\hline 50 & Sex + COVID 19 & $\begin{array}{l}\text { https://youtu.be/ } \\
\text { Zka6DCPYm1w }\end{array}$ & 2484 & 31.03 .2020 & 48 & 650 & 13.54 & 5 & 0 & 4 & 2 & 3 \\
\hline 51 & $\begin{array}{l}\text { A sexual intimacy guide } \\
\text { amid COVID-19 outbreak }\end{array}$ & $\begin{array}{l}\text { https://youtu.be/- } \\
\text { TF9lqtEeEU }\end{array}$ & 283 & 20.03.2020 & 60 & 668 & 11.13 & 22 & 0 & 15 & 5 & 5 \\
\hline 52 & $\begin{array}{l}\text { Update: fertility } \\
\text { treatment during } \\
\text { COVID-19 with Dr. } \\
\text { William Schoolcraft }\end{array}$ & $\begin{array}{l}\text { https://youtu. } \\
\text { be/6bgmaWoGPqg }\end{array}$ & 290 & 3.04 .2020 & 46 & 696 & 15.13 & 11 & 0 & 0 & 5 & 5 \\
\hline 53 & $\begin{array}{l}\text { How to SEX with } \\
\text { COVID-19 around II } \\
\text { Coronavirus can be } \\
\text { sexually transmitted }\end{array}$ & $\begin{array}{l}\text { https://youtu.be/ } \\
\text { CK-6r31XCX8 }\end{array}$ & 321 & 27.03.2020 & 53 & 541 & 10.2 & 4 & 0 & 0 & 5 & 5 \\
\hline 54 & $\begin{array}{l}\text { COVID-19 puts fertility } \\
\text { treatment, family's } \\
\text { dreams on hold }\end{array}$ & $\begin{array}{l}\text { https://youtu.be/ } \\
\text { LMel00ck18g }\end{array}$ & 117 & 11.04 .2020 & 26 & 699 & 26.88 & 3 & 0 & 0 & 3 & 4 \\
\hline 55 & $\begin{array}{l}\text { Handling infertility } \\
\text { treatments during the } \\
\text { coronavirus pandemic }\end{array}$ & $\begin{array}{l}\text { https://youtu.be/ } \\
\text { PSojdfFcEh4 }\end{array}$ & 136 & 24.03 .2020 & 26 & 685 & 26.34 & 5 & 0 & 0 & 4 & 5 \\
\hline 56 & $\begin{array}{l}\text { Semen retention|This } \\
\text { is your last chance } \\
\text { (COVID-19) }\end{array}$ & $\begin{array}{l}\text { https://youtu.be/ } \\
\text { ePDU0_tfzjo }\end{array}$ & 411 & 25.03 .2020 & 55 & 455 & 8.27 & 26 & 3 & 11 & 2 & 2 \\
\hline 57 & $\begin{array}{l}\text { Greater Boston Urology } \\
\text { addresses COVID-19 } \\
\text { pandemic }\end{array}$ & $\begin{array}{l}\text { https://youtu.be/ } \\
\text { YBZDhfYI7VU }\end{array}$ & 205 & 31.03 .2020 & 49 & 430 & 8.77 & 4 & 0 & 0 & 4 & 4 \\
\hline 58 & $\begin{array}{l}\text { How is COVID-19 } \\
\text { affecting people with } \\
\text { kidney failure and on the } \\
\text { transplant waiting list? }\end{array}$ & $\begin{array}{l}\text { https://youtu. } \\
\text { be/_RIUt0hq8p0 }\end{array}$ & 925 & 28.04.2020 & 51 & 417 & 8.17 & 5 & 1 & 0 & 4 & 4 \\
\hline 59 & $\begin{array}{l}\text { Bladder cancer, BCG and } \\
\text { COVID-19|Treatment } \\
\text { vaccine update for } \\
\text { patients and families }\end{array}$ & $\begin{array}{l}\text { https://youtu. } \\
\text { be/8R6UQtejVuw } \\
\text { ?list=PL6k8gH } \\
\text { Lg nM67FCo } \\
\text { xlgRYOsvl } \\
\text { E0vmudson }\end{array}$ & 3107 & 24.04 .2020 & 25 & 403 & 7.19 & 3 & 0 & 1 & 5 & 5 \\
\hline 60 & $\begin{array}{l}\text { Semen } \\
\text { retention|COVID-19 Your } \\
\text { immune system NEEDS } \\
\text { YOU }\end{array}$ & $\begin{array}{l}\text { https://youtu. } \\
\text { be/8KzLnH-bmxE }\end{array}$ & 582 & 19.03 .2020 & 61 & 386 & 6.32 & 44 & 0 & 6 & 2 & 2 \\
\hline 61 & $\begin{array}{l}\text { Kidney transplants and } \\
\text { COVID-19|National } \\
\text { Kidney Foundation }\end{array}$ & $\begin{array}{l}\text { https://youtu.be/ } \\
\text { HFA-BERo0a0 }\end{array}$ & 50 & 21.04.2020 & 28 & 416 & 14.85 & 5 & 0 & 1 & 4 & 4 \\
\hline 62 & $\begin{array}{l}\text { Coronavirus (COVID-19) } \\
\text { Impact on IVF and egg } \\
\text { freezing process|RSMC }\end{array}$ & $\begin{array}{l}\text { https://youtu.be/ } \\
\text { OYmXkXAnOvM }\end{array}$ & 183 & 17.03.2020 & 63 & 361 & 5.73 & 4 & 1 & 3 & 4 & 5 \\
\hline 63 & $\begin{array}{l}\text { Are kidney donors at } \\
\text { greater risk for COVID- } \\
\text { 19?|National Kidney } \\
\text { Foundation }\end{array}$ & $\begin{array}{l}\text { https://youtu.be/ } \\
\text { XxADQT7W2yc }\end{array}$ & 63 & 22.04 .2020 & 27 & 321 & 11.88 & 3 & 0 & 1 & 3 & 4 \\
\hline 64 & $\begin{array}{l}\text { Infertility \& COVID-19 } \\
\text { Session 2 / www.gbrclinic. } \\
\text { com/+91-9940105555 }\end{array}$ & $\begin{array}{l}\text { https://youtu.be/ } \\
\text { cgJ2Xyo2Eel }\end{array}$ & 2234 & 23.04 .2020 & 26 & 293 & 11.26 & 3 & 0 & 0 & 3 & 4 \\
\hline 65 & $\begin{array}{l}\text { Dr. Anindita Singh } \\
\text { on fertility treatment } \\
\text { plans during } \\
\text { COVID-19 pandemic. } \\
\text { Teleconsult@1800 } 313 \\
5677\end{array}$ & $\begin{array}{l}\text { https://youtu.be/ } \\
\text { iMW4x8ZKGGQ }\end{array}$ & 172 & 30.03 .2020 & 50 & 297 & 5.94 & 11 & 0 & 0 & 3 & 4 \\
\hline
\end{tabular}




\begin{tabular}{|c|c|c|c|c|c|c|c|c|c|c|c|c|}
\hline 66 & $\begin{array}{l}\text { Fertility treatments } \\
\text { halted, delayed during } \\
\text { COVID-19 }\end{array}$ & $\begin{array}{l}\text { https://youtu.be/ } \\
\text { OduwMOxttTg }\end{array}$ & 122 & 15.04 .2020 & 34 & 280 & 8.23 & 0 & 3 & 2 & 3 & 4 \\
\hline 67 & $\begin{array}{l}\text { CHR's precautions against } \\
\text { COVID-19 (coronavirus) } \\
\text { infections }\end{array}$ & $\begin{array}{l}\text { https://youtu.be/ } \\
\text { E4IAvKMAnUI }\end{array}$ & 917 & 23.03 .2020 & 57 & 270 & 4.73 & 8 & 0 & 5 & 5 & 5 \\
\hline 68 & $\begin{array}{l}\text { Mark's April } 2020 \text { Update } \\
\text { - Now COVID-19, too? - } \\
\text { Mark's prostate cancer } \\
\text { experience }\end{array}$ & $\begin{array}{l}\text { https://youtu.be/ } \\
\text { Icjl88ja3Ag }\end{array}$ & 391 & 13.04 .2020 & 36 & 263 & 7.3 & 32 & 1 & 4 & 3 & 4 \\
\hline 69 & $\begin{array}{l}\text { ICS - COVID } 19 \text { and } \\
\text { management in } \\
\text { neurogenic bladder }\end{array}$ & $\begin{array}{l}\text { https://youtu.be/ } \\
\text { CbAWK1NRLZY }\end{array}$ & 208 & 17.04 .2020 & 32 & 264 & 8.25 & 2 & 0 & 0 & 5 & 5 \\
\hline 70 & $\begin{array}{l}\text { Fertility treatment during } \\
\text { COVID-19 with Dr. } \\
\text { William Schoolcraft }\end{array}$ & $\begin{array}{l}\text { https://youtu.be/ } \\
\text { TDX20fTo4 m0 }\end{array}$ & 163 & 27.03.2020 & 53 & 253 & 4.77 & 0 & 0 & 0 & 4 & 4 \\
\hline 71 & $\begin{array}{l}\text { Dr Ritu Hinduja - Advise } \\
\text { for patients on fertility } \\
\text { treatment during } \\
\text { COVID-19 pandemic }\end{array}$ & $\begin{array}{l}\text { https://youtu. } \\
\text { be/_KcjkVmFMKg }\end{array}$ & 121 & 28.03.2020 & 52 & 250 & 4.8 & 5 & 0 & 1 & 4 & 5 \\
\hline 72 & $\begin{array}{l}\text { Ovarian stimulation } \\
\text { during COVID- } 19\end{array}$ & $\begin{array}{l}\text { https://youtu.be/ } \\
\text { SdDcDDSBOUY }\end{array}$ & 95 & 6.04 .2020 & 43 & 246 & 5.72 & 7 & 1 & 0 & 4 & 5 \\
\hline 73 & $\begin{array}{l}\text { Fertility patients face } \\
\text { challenges during } \\
\text { COVID-19 pandemic }\end{array}$ & $\begin{array}{l}\text { https://youtu.be/ } \\
\text { jJDq0_Uhhmw }\end{array}$ & 106 & 10.04 .2020 & 39 & 231 & 5.92 & 3 & 0 & 1 & 3 & 4 \\
\hline 74 & $\begin{array}{l}\text { COVID-19 North East } \\
\text { United States: What } \\
\text { you should know about } \\
\text { bladder cancer and } \\
\text { COVID-19 }\end{array}$ & $\begin{array}{l}\text { https://youtu.be/ } \\
\text { BkOTMKOkC1g }\end{array}$ & 3385 & 9.04 .2020 & 40 & 227 & 5.67 & 1 & 0 & 0 & 5 & 5 \\
\hline 75 & $\begin{array}{l}\text { Telehealth urology visits } \\
\text { and COVID-19 updates } \\
\text { ACU }\end{array}$ & $\begin{array}{l}\text { https://youtu.be/ } \\
\text { xWLN98azf2s }\end{array}$ & 407 & 26.03 .2020 & 54 & 214 & 3.96 & 12 & 0 & 4 & 5 & 5 \\
\hline 76 & $\begin{array}{l}\text { Infertility treatment } \\
\text { during COVID-19|Dr. } \\
\text { Akriti Bharati|Vatsalya } \\
\text { Natural IVF|Best IVF Clinic }\end{array}$ & $\begin{array}{l}\text { https://youtu.be/ } \\
\text { barz3nR2VMg }\end{array}$ & 216 & 24.04 .2020 & 25 & 227 & 9.08 & 9 & 0 & 1 & 4 & 4 \\
\hline 77 & $\begin{array}{l}\text { IVF \& coronavirus: } \\
\text { treatments suspended } \\
\text { during pandemic }\end{array}$ & $\begin{array}{l}\text { https://youtu.be/ } \\
\text { UXkXbl8b4hw }\end{array}$ & 99 & 7.04.2020 & 42 & 201 & 4.78 & 3 & 0 & 1 & 4 & 5 \\
\hline 78 & $\begin{array}{l}\text { COVID } 19 \text { and fertility } \\
\text { treatment \#IVFWEBINARS }\end{array}$ & $\begin{array}{l}\text { https://youtu.be/ } \\
\text { clBYFJPparA }\end{array}$ & 3896 & 7.04.2020 & 44 & 222 & 5.04 & 1 & 0 & 0 & 5 & 5 \\
\hline 79 & $\begin{array}{l}\text { How to enjoy sex and to } \\
\text { avoid spreading COVID- } \\
\text { 19??|COVID-19|My } \\
\text { Biography }\end{array}$ & $\begin{array}{l}\text { https://youtu.be/ } \\
\text { uNB2Y4CVwnM }\end{array}$ & 72 & 24.03.2020 & 58 & 196 & 3.37 & 1 & 0 & 0 & 3 & 3 \\
\hline 80 & $\begin{array}{l}\text { Thinking about fertility } \\
\text { treatment during } \\
\text { COVID-19 }\end{array}$ & $\begin{array}{l}\text { https://youtu.be/ } \\
\text { PUuhf04f0s4 }\end{array}$ & 223 & 15.04 .2020 & 36 & 191 & 5.3 & 2 & 0 & 0 & 4 & 5 \\
\hline 81 & $\begin{array}{l}\text { COVID-19 Southeast } \\
\text { United States: what } \\
\text { you should know about } \\
\text { bladder cancer and } \\
\text { COVID-19 }\end{array}$ & $\begin{array}{l}\text { https://youtu.be/ } \\
\text { blv7IVqUIVk }\end{array}$ & 3585 & 10.04 .2020 & 42 & 173 & 4.11 & 4 & 0 & 0 & 5 & 5 \\
\hline 82 & $\begin{array}{l}\text { Dubai OBGYN explains } \\
\text { infertility procedures } \\
\text { during COVID-19 }\end{array}$ & $\begin{array}{l}\text { https://youtu. } \\
\text { be/9NIXgoeOh0o }\end{array}$ & 283 & 4.05 .2020 & 17 & 171 & 10.05 & 0 & 0 & 1 & 4 & 5 \\
\hline 83 & $\begin{array}{l}\text { COVID-19 and the West } \\
\text { Coast: what you should } \\
\text { know about COVID-19 } \\
\text { and bladder cancer }\end{array}$ & $\begin{array}{l}\text { https://youtu.be/ } \\
\text { EqW220vwcRE }\end{array}$ & 3471 & 14.04 .2020 & 38 & 159 & 4.18 & 3 & 0 & 0 & 5 & 5 \\
\hline
\end{tabular}




\begin{tabular}{|c|c|c|c|c|c|c|c|c|c|c|c|c|}
\hline 84 & $\begin{array}{l}\text { Covid } 19 \text { could damage } \\
\text { male fertility?|Male } \\
\text { Sex|Sex Life|Sex } \\
\text { Education|Research } \\
\text { Associate }\end{array}$ & $\begin{array}{l}\text { https://youtu.be/ } \\
\text { INszKIxC3vw }\end{array}$ & 121 & 13.03 .2020 & 70 & 154 & 2.2 & 8 & 0 & 3 & 3 & 4 \\
\hline 85 & $\begin{array}{l}\text { Can COVID19 } \\
\text { (Coronavirus) cause } \\
\text { INFERTILITY? Long lasting } \\
\text { impacts of COVID- } \\
\text { 19|Bite-size Science }\end{array}$ & $\begin{array}{l}\text { https://youtu.be/ } \\
\text { KzU06gs37ts }\end{array}$ & 202 & 24.04 .2020 & 28 & 146 & 5.21 & 17 & 0 & 19 & 5 & 5 \\
\hline 86 & $\begin{array}{l}\text { Safer sex in the time of } \\
\text { COVID } 19\end{array}$ & $\begin{array}{l}\text { https://youtu. } \\
\text { be/8off_2wtdAw }\end{array}$ & 198 & 3.04 .2020 & 49 & 125 & 2.55 & 6 & 0 & 1 & 3 & 4 \\
\hline 87 & $\begin{array}{l}\text { What COVID-19 } \\
\text { precautions mean for } \\
\text { patients going through } \\
\text { fertility treatment|Your } \\
\text { Morning }\end{array}$ & $\begin{array}{l}\text { https://youtu. } \\
\text { be/-_MwPDTUKVI }\end{array}$ & 205 & 24.03.2020 & 59 & 124 & 2.1 & 1 & 0 & 1 & 4 & 5 \\
\hline 88 & $\begin{array}{l}\text { Fertility Clinic in Orange } \\
\text { County response to } \\
\text { Coronavirus (COVID-19) }\end{array}$ & $\begin{array}{l}\text { https://youtu.be/ } \\
\text { q08ITJ3 mwbl }\end{array}$ & 77 & 19.03 .2020 & 64 & 121 & 1.89 & 1 & 0 & 0 & 3 & 3 \\
\hline 89 & $\begin{array}{l}\text { COVID-19 and Infertility: } \\
\text { what the coronavirus } \\
\text { pandemic means for } \\
\text { patients of CARE Fertility }\end{array}$ & $\begin{array}{l}\text { https://youtu.be/ } \\
\text { IKDHI9ybAUY }\end{array}$ & 228 & 23.03.2020 & 60 & 109 & 1.81 & 3 & 0 & 0 & 4 & 5 \\
\hline 90 & $\begin{array}{l}\text { REPORT: Coronavirus may } \\
\text { damage testicles }\end{array}$ & $\begin{array}{l}\text { https://youtu.be/ } \\
\text { uf2eMseatYg }\end{array}$ & 332 & 6.04 .2020 & 46 & 107 & 2.32 & 6 & 0 & 0 & 5 & 5 \\
\hline 91 & $\begin{array}{l}\text { Utah researchers say } \\
\text { COVID-19 unlikely to } \\
\text { spread through sexual } \\
\text { intercourse }\end{array}$ & $\begin{array}{l}\text { https://youtu.be/ } \\
\text { laEuq-AlOqE }\end{array}$ & 474 & 29.04.2020 & 23 & 106 & 4.6 & 12 & 1 & 4 & 4 & 4 \\
\hline 92 & $\begin{array}{l}\text { Facebook Live - bladder } \\
\text { cancer and staying } \\
\text { grounded in a time of } \\
\text { crisis }\end{array}$ & $\begin{array}{l}\text { https://youtu.be/ } \\
\text { OP3AnQeVBOY }\end{array}$ & 3204 & 20.04 .2020 & 32 & 98 & 3.06 & 1 & 0 & 0 & 5 & 5 \\
\hline 93 & $\begin{array}{l}\text { Doctor: IVF treatments } \\
\text { are getting delayed due } \\
\text { to COVID-19 }\end{array}$ & $\begin{array}{l}\text { https://youtu. } \\
\text { be/8XCidyaGXHO }\end{array}$ & 1402 & 9.04 .2020 & 43 & 92 & 2.13 & 0 & 1 & 0 & 4 & 5 \\
\hline 94 & $\begin{array}{l}\text { Coronavirus and sex: does } \\
\text { sex transmit COVID-19? } \\
\text { How can you protect } \\
\text { yourself while having sex? }\end{array}$ & $\begin{array}{l}\text { https://youtu.be/ } \\
\text { h05RaLbHYOw }\end{array}$ & 1006 & 10.04 .2020 & 42 & 89 & 2.11 & 1 & 0 & 0 & 4 & 4 \\
\hline 95 & $\begin{array}{l}\text { QctA: Prostate Cancer \&t } \\
\text { COVID-19 }\end{array}$ & $\begin{array}{l}\text { https://youtu.be/ } \\
\text { zJhkdf15Rtc }\end{array}$ & 1088 & 1.05 .2020 & 20 & 91 & 4.55 & 3 & 0 & 0 & 5 & 5 \\
\hline 96 & $\begin{array}{l}\text { COVID-19: Kidney } \\
\text { Cancer Awareness Month } \\
\text { Facebook Live O\&tA }\end{array}$ & $\begin{array}{l}\text { https://youtu.be/ } \\
\text { Gc0AyXI4jWs }\end{array}$ & 798 & 18.03.2020 & 65 & 80 & 1.23 & 1 & 0 & 0 & 5 & 5 \\
\hline 97 & $\begin{array}{l}\text { COVID } 19 \text { and the Central } \\
\text { US: What you should } \\
\text { know about bladder } \\
\text { cancer and COVID-19 }\end{array}$ & $\begin{array}{l}\text { https://youtu. } \\
\text { be/6nFOhcnbKr8 }\end{array}$ & 3594 & 22.04.2020 & 30 & 82 & 2.73 & 0 & 0 & 0 & 5 & 5 \\
\hline 98 & $\begin{array}{l}\text { Coronavirus-Part 1- } \\
\text { Prostate cancer and } \\
\text { COVID-19 }\end{array}$ & $\begin{array}{l}\text { https://youtu.be/ } \\
\text { qtt-AvTtS5s }\end{array}$ & 417 & 24.03 .2020 & 59 & 82 & 1.38 & 1 & 0 & 0 & 4 & 5 \\
\hline 99 & $\begin{array}{l}\text { Parents struggling with } \\
\text { infertility in limbo due to } \\
\text { COVID-19 crisis }\end{array}$ & $\begin{array}{l}\text { https://youtu.be/ } \\
\text { RGa8AxXTyVY }\end{array}$ & 152 & 1.04 .2020 & 51 & 75 & 1.47 & 0 & 1 & 0 & 4 & 5 \\
\hline 100 & $\begin{array}{l}\text { How are urologists } \\
\text { handling COVID-19? }\end{array}$ & $\begin{array}{l}\text { https://youtu.be/ } \\
\text { r11IHtakZBO }\end{array}$ & 88 & 6.04 .2020 & 46 & 82 & 1.78 & 1 & 1 & 0 & 4 & 5 \\
\hline
\end{tabular}




\begin{tabular}{|c|c|c|c|c|c|c|c|c|c|c|c|c|}
\hline 101 & $\begin{array}{l}\text { COVID-19: What the } \\
\text { virus means for infertility } \\
\text { patients }\end{array}$ & $\begin{array}{l}\text { https://youtu.be/ } \\
\text { yXRtP_-OnPw }\end{array}$ & 1103 & 25.03 .2020 & 58 & 71 & 1.22 & 2 & 0 & 0 & 4 & 5 \\
\hline 102 & $\begin{array}{l}\text { Calming fears about } \\
\text { COVID-19 \& Your Fertility } \\
\text { With Dr. Serena H. Chen \& } \\
\text { Medanswers }\end{array}$ & $\begin{array}{l}\text { https://youtu.be/ } \\
\text { Wk1yAxaaH4I }\end{array}$ & 377 & 16.03.2020 & 67 & 69 & 1.02 & 2 & 0 & 1 & 4 & 5 \\
\hline 103 & $\begin{array}{l}\text { Coping with anxiety } \\
\text { during infertility and } \\
\text { COVID-19 }\end{array}$ & $\begin{array}{l}\text { https://youtu.be/t- } \\
\text { AzOnq9lzc }\end{array}$ & 278 & 16.04 .2020 & 36 & 77 & 2.13 & 1 & 0 & 0 & 4 & 5 \\
\hline 104 & $\begin{array}{l}\text { PCFNZ Webinar|The } \\
\text { practicalities of living } \\
\text { with prostate cancer } \\
\text { during COVID } 19\end{array}$ & $\begin{array}{l}\text { https://youtu.be/ } \\
\text { exji60p0mxo }\end{array}$ & 3070 & 23.04 .2020 & 29 & 69 & 2.37 & 1 & 0 & 0 & 5 & 5 \\
\hline 105 & $\begin{array}{l}\text { Corona and kidney } \\
\text { transplant in } 2020\end{array}$ & $\begin{array}{l}\text { https://youtu.be/ } \\
\text { mtwLs2_4cTU }\end{array}$ & 101 & 27.03.2020 & 56 & 53 & 0.94 & 4 & 0 & 1 & 3 & 5 \\
\hline 106 & $\begin{array}{l}\text { Fertility and the impact of } \\
\text { COVID-19 }\end{array}$ & $\begin{array}{l}\text { https://youtu.be/ } \\
\text { ETVbKA5WL00 }\end{array}$ & 576 & 30.03 .2020 & 53 & 49 & 0.92 & 1 & 0 & 1 & 4 & 5 \\
\hline 107 & & $\begin{array}{l}\text { This video is no } \\
\text { longer available } \\
\text { because the } \\
\text { YouTube account } \\
\text { associated with } \\
\text { this video has } \\
\text { been terminated }\end{array}$ & 67 & 17.03 .2020 & 66 & 48 & 0.72 & 0 & 1 & 0 & 3 & 3 \\
\hline 108 & $\begin{array}{l}\text { What are my prostate } \\
\text { cancer treatment options? }\end{array}$ & $\begin{array}{l}\text { https://youtu.be/ } \\
\text { Ej_mRj226EE }\end{array}$ & 111 & 10.04 .2020 & 42 & 48 & 1.14 & 0 & 1 & 0 & 3 & 4 \\
\hline 109 & $\begin{array}{l}\text { What are the benefits of } \\
\text { telemedicine? }\end{array}$ & $\begin{array}{l}\text { https://youtu.be/ } \\
\text { I8Hy48-rnmg }\end{array}$ & 136 & 7.04.2020 & 45 & 49 & 1.08 & 1 & 1 & 0 & 3 & 4 \\
\hline 110 & $\begin{array}{l}\text { STRESS, FERTILITY \& } \\
\text { COVID-19 WEBINAR \#2 }\end{array}$ & $\begin{array}{l}\text { https://youtu.be/ } \\
\text { Ttekwwl7SfE }\end{array}$ & 960 & 7.04.2020 & 45 & 48 & 1.06 & 0 & 0 & 0 & 4 & 5 \\
\hline 111 & $\begin{array}{l}\text { COVID-19 and the } \\
\text { South West US: What } \\
\text { you should know about } \\
\text { bladder cancer and } \\
\text { COVID-19 }\end{array}$ & $\begin{array}{l}\text { https://youtu.be/ } \\
\text { R8IRF3csu_8 }\end{array}$ & 3872 & 15.04 .2020 & 37 & 46 & 1.24 & 0 & 0 & 0 & 5 & 5 \\
\hline 112 & $\begin{array}{l}\text { Managing IVF pregnancy } \\
\text { during COVID-19 - Dr. } \\
\text { Nidhi Jha - Southend } \\
\text { Fertility \& IVF }\end{array}$ & $\begin{array}{l}\text { https://youtu.be/ } \\
\text { rFNKVoD20IU }\end{array}$ & 81 & 10.04 .2020 & 42 & 38 & 0.9 & 0 & 0 & 0 & 3 & 4 \\
\hline 113 & $\begin{array}{l}\text { Will COVID } 19 \text { affects } \\
\text { male infertility? }\end{array}$ & $\begin{array}{l}\text { https://youtu.be/ } \\
\text { shgNcT95eYM }\end{array}$ & 112 & 19.04.2020 & 31 & 37 & 1.19 & 1 & 0 & 0 & 3 & 4 \\
\hline 114 & What is telemedicine? & $\begin{array}{l}\text { https://youtu.be/ } \\
\text { clnijuusINc }\end{array}$ & 55 & 7.04.2020 & 45 & 32 & 0.71 & 0 & 1 & 0 & 3 & 4 \\
\hline 115 & $\begin{array}{l}\text { Does Medicare/insurance } \\
\text { cover telemedicine? }\end{array}$ & $\begin{array}{l}\text { https://youtu.be/ } \\
\text { i6809V11--c }\end{array}$ & 84 & 10.04 .2020 & 42 & 24 & 0.57 & 0 & 1 & 0 & 3 & 4 \\
\hline 116 & $\begin{array}{l}\text { Keeping sex and desire } \\
\text { burning during COVID } \\
\text { feat Alexa Martinez }\end{array}$ & $\begin{array}{l}\text { https://youtu.be/ } \\
\text { aLWA-oG4jvl }\end{array}$ & 3407 & 6.04 .2020 & 46 & 21 & 0.45 & 1 & 0 & 2 & 2 & 3 \\
\hline 117 & $\begin{array}{l}\text { Do I have to be tech- } \\
\text { savvy for a virtual visit? }\end{array}$ & $\begin{array}{l}\text { https://youtu.be/ } \\
\text { 0j6P0x80aws }\end{array}$ & 93 & 10.04 .2020 & 42 & 12 & 0.28 & 0 & 1 & 0 & 3 & 4 \\
\hline 118 & $\begin{array}{l}\text { Managing urology } \\
\text { conditions during } \\
\text { COVID-19 }\end{array}$ & $\begin{array}{l}\text { https://youtu.be/ } \\
\text { Uufc16qXuFM }\end{array}$ & 946 & 29.04 .2020 & 21 & 14 & 0.66 & 0 & 0 & 0 & 4 & 5 \\
\hline 119 & $\begin{array}{l}\text { COVID-19: infertility } \\
\text { consultations during } \\
\text { pandemic }\end{array}$ & $\begin{array}{l}\text { https://youtu.be/ } \\
\text { fs66ySIcoNk }\end{array}$ & 507 & 22.04 .2020 & 28 & 15 & 0.53 & 0 & 0 & 0 & 4 & 5 \\
\hline
\end{tabular}




\begin{tabular}{|c|c|c|c|c|c|c|c|c|c|c|c|c|}
\hline 120 & $\begin{array}{l}\text { Renal medullary } \\
\text { carcinoma and COVID-19: } \\
\text { Protecting patients with } \\
\text { advanced cancers }\end{array}$ & $\begin{array}{l}\text { https://youtu.be/- } \\
\text { 1GRPtDMDUc }\end{array}$ & 183 & 19.04.2020 & 31 & 6 & 0.19 & 0 & 0 & 0 & 4 & 5 \\
\hline 121 & $\begin{array}{l}\text { COVID-19 and prostate } \\
\text { cancer patients }\end{array}$ & $\begin{array}{l}\text { https://youtu.be/ } \\
\text { YCbOEAulAOM }\end{array}$ & 115 & 24.04 .2020 & 26 & 7 & 0.26 & 0 & 0 & 0 & 4 & 5 \\
\hline 122 & $\begin{array}{l}\text { RCC in the COVID era: } \\
\text { immunotherapy for } \\
\text { metastatic cancer }\end{array}$ & $\begin{array}{l}\text { https://youtu.be/ } \\
\text { mypLExMze7A }\end{array}$ & 664 & 14.04 .2020 & 36 & 4 & 0.11 & 0 & 0 & 0 & 5 & 5 \\
\hline 123 & $\begin{array}{l}\text { Explaining adapted IVF } \\
\text { protocols during COVID- } \\
\text { 19|CARE Fertility }\end{array}$ & $\begin{array}{l}\text { https://youtu.be/ } \\
\text { JaltLP22R4o }\end{array}$ & 172 & 15.05 .2020 & 7 & 125 & 17.85 & 2 & 0 & 0 & 4 & 5 \\
\hline 124 & $\begin{array}{l}\text { COVID safety protocol at } \\
\text { Nova IVF fertility }\end{array}$ & $\begin{array}{l}\text { https://youtu. } \\
\text { be/2IU_do4sb0o }\end{array}$ & 327 & 21.05 .2020 & 1 & 68 & 68 & 2 & 0 & 0 & 4 & 5 \\
\hline 125 & $\begin{array}{l}\text { How to keep safe from } \\
\text { the coronavirus after your } \\
\text { IVF treatment resumes }\end{array}$ & $\begin{array}{l}\text { https://youtu.be/ } \\
\text { kI3MmMu4Xu0 }\end{array}$ & 313 & 14.05 .2020 & 8 & 819 & 102.37 & 37 & 0 & 11 & 5 & 5 \\
\hline 126 & $\begin{array}{l}\text { The new normal, adjusting } \\
\text { to COVID-19 Webinar }\end{array}$ & $\begin{array}{l}\text { https://youtu.be/ } \\
\text { ZBhkdonqeig }\end{array}$ & 3690 & 17.05 .2020 & 5 & 23 & 4.6 & 2 & 0 & 0 & 4 & 5 \\
\hline 127 & $\begin{array}{l}\text { How has COVID-19 } \\
\text { affected ongoing IVF } \\
\text { treatment|Dr. Shweta } \\
\text { Goswami|Zeeva Fertility } \\
\text { Clinic }\end{array}$ & $\begin{array}{l}\text { https://youtu.be/ } \\
\text { ven7Mwt5i_Y }\end{array}$ & 419 & 16.05 .2020 & 6 & 15 & 2.5 & 0 & 0 & 0 & 4 & 5 \\
\hline 128 & $\begin{array}{l}\text { Veterans, Prostate Cancer } \\
\text { \& COVID-19 }\end{array}$ & $\begin{array}{l}\text { https://youtu.be/ } \\
\text { wSRIJ92Chxg }\end{array}$ & 933 & 6.05 .2020 & 16 & 27 & 1.68 & 1 & 0 & 0 & 4 & 5 \\
\hline 129 & $\begin{array}{l}\text { Mark Hagenbuch: Battling } \\
\text { prostate cancer during } \\
\text { COVID-19 crisis }\end{array}$ & $\begin{array}{l}\text { https://youtu. } \\
\text { be/5NjRFb009-4 }\end{array}$ & 708 & 7.05.2020 & 15 & 14 & 0.93 & 0 & 0 & 0 & 4 & 5 \\
\hline 130 & $\begin{array}{l}\text { Yoga for prostate cancer } \\
\text { Et COVID-19 relaxation }\end{array}$ & $\begin{array}{l}\text { https://youtu. } \\
\text { be/4hnUK6PmDg0 }\end{array}$ & 1869 & 6.05 .2020 & 15 & 7 & 0.46 & 0 & 0 & 0 & 3 & 4 \\
\hline 131 & $\begin{array}{l}\text { Prostate cancer, COVID-19 } \\
\text { \& stress: Emotional } \\
\text { check-in time }\end{array}$ & $\begin{array}{l}\text { https://youtu.be/ } \\
\text { PVHAmrjX5I0 }\end{array}$ & 1901 & 7.05.2020 & 15 & 10 & 0.66 & 0 & 0 & 0 & 3 & 4 \\
\hline 132 & $\begin{array}{l}\text { ZERO's CEO: 'I have } \\
\text { COVID-19' }\end{array}$ & $\begin{array}{l}\text { https://youtu.be/ } \\
\text { LVYpnhqkY8g }\end{array}$ & 1663 & 24.04 .2020 & 27 & 166 & 6.14 & 0 & 2 & 0 & 3 & 4 \\
\hline 133 & $\begin{array}{l}\text { Eckert \& Ziegler BEBIG: } \\
\text { Prostate brachytherapy in } \\
\text { times of COVID-19 }\end{array}$ & $\begin{array}{l}\text { https://youtu.be/ } \\
\text { Qb7Lsv77Tp4 }\end{array}$ & 134 & 13.05 .2020 & 9 & 317 & 35.22 & 5 & 0 & 0 & 3 & 4 \\
\hline 134 & $\begin{array}{l}\text { What patients with } \\
\text { bladder cancer can do } \\
\text { When COVID-19 impacts } \\
\text { their care }\end{array}$ & $\begin{array}{l}\text { https://youtu.be/ } \\
\text { ixheM0Fur88 }\end{array}$ & 159 & 14.05.2020 & 8 & 27 & 3.37 & 0 & 0 & 0 & 3 & 4 \\
\hline 135 & $\begin{array}{l}\text { Sex and dating in the post } \\
\text { COVID World|Q+A }\end{array}$ & $\begin{array}{l}\text { https://youtu. } \\
\text { be/_0CvLC3Vszs }\end{array}$ & 198 & 19.05.2020 & 3 & 761 & 253.66 & 6 & 15 & 3 & 2 & 3 \\
\hline 136 & $\begin{array}{l}\text { Sexual health during the } \\
\text { COVID era }\end{array}$ & $\begin{array}{l}\text { https://youtu.be/ } \\
\text { kQiqROd_Nls }\end{array}$ & 227 & 19.05 .2020 & 3 & 18 & 6 & 1 & 0 & 0 & 2 & 3 \\
\hline
\end{tabular}

more important for these patients. Boehm et al. (20) reported that $63.2 \%$ of patients with urological conditions were eligible for telemedicine. Among eligible patients, 54.1\% stated that they would prefer telemedicine instead of face-to-face consultation. This rate was higher for those aged $<68$ years (20). Technical limitations were the main cause for refusal. Uro-oncological diseases were the most common reason among patients both eligible and willing to use telemedicine (72.2\%). Other diseases were incontinence/ bladder dysfunction (6.9\%), andrological condition (5.5\%), benign prostatic hyperplasia (3.7\%), urinary tract infection (3.2\%), urolithiasis (2.3\%) and other reasons (5.9\%) (20). However, interestingly, we observed that uploaded video rates, number of total views and number of views per day were higher for andrology-related YouTube videos. This finding implies that since andrological condition may be the topic that people want to learn most, more andrology-related videos have been uploaded to YouTube. We think that fewer videos on uro-oncology are uploaded since the approach and strategy related to uro-oncology is clearer. By contrast, we think that there are more videos on andrology and sexual life since there are more controversial ideas on this topic. Indeed, among 
all urology-related videos during the COVID-19 pandemic, the most viewed videos are related to andrology. We consider that sexual health makes people more curious. For this reason, videos about andrology may be viewed more. We also assume that non-healthcare professionals have uploaded more videos on andrology, considering that they will capture a wider audience.

Sufficient information should be provided to patients under follow-up for urological diseases. However, it appears difficult for patients to determine which of their symptoms can be deferred and for how long. In addition, andrological diseases except for penile fracture, priapism, testicular torsion and scrotal and penile traumas are mostly treated electively and the international guidelines categorise andrological pathologies as a non-essential issue, so consultation with healthcare professionals for this purpose and treatment has to be postponed during the COVID-19 period $(8,10)$. Since patients with andrological pathologies already have a low quality of life, not being able to get treatment during this period may worsen the severity of the disease (17). Because it is not very practical for a urologist to discuss these situations by telemedicine with every patient, we consider that informative, reliable and easyto-understand YouTube videos may be useful for patients and their relatives.

Despite the ease of access to information, YouTube videos containing medical advertisements and for profit are often listed among the more popular videos (22). For that reason, there is a risk of exposure to misleading and incorrect information $(5,23)$. Although some previous studies have found YouTube to be a source of accurate educational and useful health information $(7,16,24,25)$, there have been opposite views in the literature about the reliability of these videos $(16,23,26,27)$. Studies have recommended using more validated formal assessment tools such as the modified DISCERN tool, GOS or other forms [e.g. instrument that was developed by Azer et al. $(24)](6,7,16)$. However, it is not possible for lay people to make this evaluation and thereby determine the reliability and quality of the video.

Increasing the awareness of male sexual health and encouraging them to see a physician is important to improve their quality of life. Several studies have stated that the majority of YouTube videos, including videos on andrological topics such as premature ejaculation, are unreliable sources of medical information $(7,22,23,28)$. In the present study, the speaker was a for-profit physician in 7 (35\%) of the 20 videos containing debated information, while 65\% of the speakers in debated videos were not healthcare providers. We found that $85.3 \%$ of all videos contained useful information, and the useful information rate was $96.2 \%$ in "universities/professional organisations/non-profit physician/physician groups", which contained $77.2 \%$ of all videos. Although most of the YouTube videos contain unreliable information and published by individual users or medical advertisement/for-profit companies $(16,23,26,27,29)$, our findings show that conversations in the COVID-19 period have been made less by individual users, medical advertisements or for-profit companies, unlike YouTube videos on other topics. However, our findings show that videos uploaded by non-healthcare professionals with lower reliability and quality scores garnered higher views and like. We think that this situation is the result of tricks made by such people to increase the discoverability and viewership of such YouTube videos. An extremely interesting headline, cover photo or custom thumbnail, using descriptive tags and writing a concise synopsis of the video content can often trigger the viewers to click on the video. Adding links to other videos that may be relevant by the video creator may be another common trick. In our opinion, these tricks are used more by non-healthcare professionals to make their videos more popular than videos of healthcare professionals.

Although andrological disorders and infertility have to be considered low priority, the number of patients who want to find solutions for their problems is not small, and they are looking for information on what they can do during this period (30). Although it was recommended to postpone appointments related to infertility in the beginning of the COVID-19 period, in the latest version of the EAU guidelines about COVID-19, male fertility surgery is only considered a high priority when women have limited ovarian reserve or have advanced maternal age, provided that a full discussion is held with the couple (8). In our analysis, 41.9\% of all YouTube videos focused on infertility during the COVID-19 period. Current developments related to infertility are one of the most looked for topics and these conversations are very popular.

\section{Study Limitations}

To the best of our knowledge, this is the first study to assess the content, view rate, reliability and quality of YouTube videos that give information on urological disorders during the COVID-19 period. However, this study has some limitations. Firstly, the search order and number of video views may change every day because of video popularity and newly added videos. Although we recorded the videos on a playlist by snapshot analysis as in previous similar studies, it is a limitation. Secondly, not analysing non-English-language videos prevents commenting on all YouTube videos. Thirdly, we used a 5-point modified DISCERN tool and GQS to analyse videos with reference to previous studies, but their formal validation has not yet been completed. Finally, we used "covid" and "coronavirus" as search keywords with all the main terms or diseases that we considered for urology. However, there is still a possibility of missing some videos using only these terms. 


\section{Conclusion}

In this study, 85.3\% of all videos contained useful information on what should be done for urological disease during the COVID-19 period. This rate was especially higher in videos uploaded by "universities/professional organisations/non-profit physician/ physician groups". The international urology committees classify andrological disorders as a non-essential issue, so they recommended that delaying consultation and treatment during the COVID-19 period. However, our results show that the rate of using YouTube as a source of information on andrology is even higher than that of uro-oncological diseases. We think that informative, easy-to-understand YouTube videos uploaded by "universities/professional organisations/non-profit physician/ physician groups" can be used as a useful additional method to improve telemedicine, which is not very practical for reaching every patient with andrological disorders that does not require follow-up.

\section{Ethics}

Ethics Committee Approval: This article does not contain any studies with human participants or animals performed by any of the authors. Therefore, there is no need for ethical approval.

Informed Consent: This article does not contain any studies with human participants or animals performed by any of the authors. Therefore, there is no need for informed consent.

Peer-review: Externally peer-reviewed.

\section{Authorship Contributions}

Concept: I.S., N.B., Design: I.S., N.B., Data Collection or Processing: I.S., N.B., Analysis or Interpretation: I.S., N.B., Literature Search: i.S., N.B., Writing: I.S.

Conflict of Interest: No conflict of interest was declared by the authors.

Financial Disclosure: The authors declare that they have no relevant financial.

\section{References}

1. Naming the coronavirus disease (COVID-19) and the virus that causes it [Internet]. World Health Organization. 2020 [cited 29 March 2020]. Available from: https://www.who.int/emergencies/diseases/novel-coronavirus-2019/ technical-guidance/naming-the-coronavirus-disease-(covid-2019)-andthe-virus-that-causes-it; 2020. Available: 21 May 2020.

2. Ho HC, Hughes T, Bozlu M, Kadıoğlu A, Somani BK. What do urologists need to know: Diagnosis, treatment, and follow-up during COVID-19 pandemic. Turk J Urol 2020;46:169-177.

3. Ficarra V, Novara G, Abrate A, Bartoletti $R$, Crestani A, De Nunzio C, Giannarini G, Gregori A, Liguori G, Mirone V, Pavan N, Scarpa RM, Simonato A, Trombetta C, Tubaro A, Porpiglia F; Research Urology Network (RUN). Urology practice during the COVID-19 pandemic. Minerva Urol Nefrol 2020;72:369-375
4. Riccardo Campi KR, Gavazzi A, Zeng G. COVID-19 and Urology - BJUI [Internet]. British Journal of Urology International. 2020 [cited 28 March 2020]. Available from: https://www.bjuinternational.com/bjui-blog/covid19-and-urology/.Available: 21 May 2020.

5. Carneiro B, Dizon DS. Prostate Cancer Social Media: In YouTube We Trust? Eur Urol 2019;75:568-569.

6. Esen E, Aslan M, Sonbahar BÇ, Kerimoğlu RS. YouTube English videos as a source of information on breast self-examination. Breast Cancer Res Treat 2019;173:629-635.

7. Gul M, Diri MA. YouTube as a Source of Information About Premature Ejaculation Treatment. J Sex Med 2019;16:1734-1740.

8. Ribal MJ, Cornford $P$, Briganti $A$, Knoll T, Gravas $S$, Babjuk M, Harding $C$ Breda A, Bex A; GORRG Group, Rassweiler JJ, Gözen AS, Pini G, Liatsikos E, Giannarini G, Mottrie A, Subramaniam R, Sofikitis N, Rocco BMC, Xie LP, Witjes JA, Mottet N, Ljungberg B, Rouprêt M, Laguna MP, Salonia A, Bonkat G, Blok BFM, Türk C, Radmayr C, Kitrey ND, Engeler DS, Lumen N, Hakenberg OW, Watkin N, Hamid R, Olsburgh J, Darraugh J, Shepherd R, Smith EJ, Chapple CR, Stenzl A, Van Poppel H, Wirth M, Sønksen J, N'Dow J. EAU Section Offices and the EAU Guidelines Panels. European Association of Urology Guidelines Office Rapid Reaction Group: An Organisationwide Collaborative Effort to Adapt the European Association of Urology Guidelines Recommendations to the Coronavirus Disease 2019 Era. Eur Urol 2020;78:21-28.

9. Mottrie A. ERUS (EAU Robotic Urology Section) guidelines during COVID-19 emergency. https://uroweb.org/wp-content/uploads/ERUS-guidelines-forCOVID-def.pdf Available: 21 May 2020.

10. Coronavirus Disease 2019 AUA Information Center https://www.auanet.org/ covid-19-info-center/covid-19-info-center Available: 21 May 2020.

11. ASRM Issues New Guidance on Fertility Care During COVID-19 Pandemic: Calls for Suspension of Most Treatments https://www.asrm.org/news-andpublications/news-and-research/press-releases-and-bulletins/asrm-issuesnew-guidance-on-fertility-care-during-covid-19-pandemiccalls-forsuspension-of-most-treatments/ Available: 21 May 2020.

12. ESHRE COVID-19 Working Group. ESHRE guidance on recommencing ART treatments. https://www.eshre.eu/Home/COVID19WG. Available: 21 May 2020.

13. Nimavat N, Singh S, Fichadiya N, Sharma P, Patel N, Kumar M, Chauhan G, Pandit N. Online Medical Education in India - Different Challenges and Probable Solutions in the Age of COVID-19. Adv Med Educ Pract 2021:12:237-243.

14. Charnock D, Shepperd S, Needham G, Gann R. DISCERN: an instrument for judging the quality of written consumer health information on treatment choices. J Epidemiol Community Health 1999;53:105-111.

15. Azer SA. Are DISCERN and JAMA Suitable Instruments for Assessing YouTube Videos on Thyroid Cancer? Methodological Concerns. J Cancer Educ 2020;5:1267-1277.

16. Selvi I, Baydilli N, Akinsal EC. Can YouTube English Videos Be Recommended as an Accurate Source for Learning About Testicular Self-examination? Urology 2020;145:181-189.

17. Rizzo M, Liguori G, Verze P, Palumbo F, Cai T, Palmieri A. How the andrological sector suffered from the dramatic Covid 19 outbreak in Italy: supportive initiatives of the Italian Association of Andrology (SIA). Int J Impot Res 2020;32:547-548.

18. Connor MJ, Winkler M, Miah S. COVID-19 pandemic - is virtual urology clinic the answer to keeping the cancer pathway moving? BJU Int 2020;125:3-4.

19. Luciani LG, Mattevi D, Cai T, Giusti G, Proietti S, Malossini G. Teleurology in the Time of Covid-19 Pandemic: Here to Stay? Urology 2020;140:4-6.

20. Boehm K, Ziewers S, Brandt MP, Sparwasser P, Haack M, Willems F, Thomas A, Dotzauer R, Höfner T, Tsaur I, Haferkamp A, Borgmann H. Telemedicine 
Online Visits in Urology During the COVID-19 Pandemic-Potential, Risk Factors, and Patients' Perspective. Eur Urol 2020;78:16-20.

21. Finazzi Agrò E, Farullo G, Balzarro $M$, Del Popolo G, Giannantoni A, Herms A, Li Marzi V, Musco S, Giammò A, Costantini E. Triage of functional, female and neuro-urology patients during and immediately after the Covid-19 outbreak. Minerva Urol Nefrol 2020;72:513-515.

22. Amante DJ, Hogan TP, Pagoto SL, English TM, Lapane KL. Access to care and use of the Internet to search for health information: results from the US National Health Interview Survey. J Med Internet Res 2015;17:106.

23. Loeb S, Sengupta $S$, Butaney M, Macaluso JN Jr, Czarniecki SW, Robbins R, Braithwaite RS, Gao L, Byrne N, Walter D, Langford A. Dissemination of Misinformative and Biased Information about Prostate Cancer on YouTube. Eur Urol 2019;75:564-567.

24. Azer SA, Bokhari RA, AISaleh GS, Alabdulaaly MM, Ateeq KI, Guerrero APS, Azer S. Experience of parents of children with autism on YouTube: are there educationally useful videos? Inform Health Soc Care 2018;43:219-233.

25. Azer SA, Aleshaiwi SM, Algrain HA, Alkhelaif RA. Nervous system examination on YouTube. BMC Med Educ 2012;12:126.
26. Farag $M$, Bolton $D$, Lawrentschuk N. Use of YouTube as a Resource for Surgical Education-Clarity or Confusion. Eur Urol Focus 2020;6:445-449.

27. Madathil KC, Rivera-Rodriguez AJ, Greenstein JS, Gramopadhye AK. Healthcare information on YouTube: A systematic review. Health Informatics J 2015;21:173-194.

28. Tanwar R, Khattar N, Sood R, Makkar A. Benign prostatic hyperplasia related content on YouTube: unregulated and concerning. Recenti Prog Med 2015;106:337-341.

29. Borgmann H, Salem J, Baunacke M, Boehm K, Groeben C, Schmid M, Siegel FP, Huber J. Mapping the landscape of urology: A new media-based crosssectional analysis of public versus academic interest. Int J Urol 2018;25:421428.

30. Esteves SC, Lombardo F, Garrido N, Alvarez J, Zini A, Colpi GM, KirkmanBrown J, Lewis SEM, Björndahl L, Majzoub A, Cho CL, Vendeira P, Hallak J, Amar E, Cocuzza M, Bento FC, Figueira RC, Sciorio R, Laursen RJ, Metwalley AM, Jindal SK, Parekattil S, Ramasamy R, Alviggi C, Humaidan P, Yovich JL, Agarwal A. SARS-CoV-2 pandemic and repercussions for male infertility patients: A proposal for the individualized provision of andrological services. Andrology 2021;9:10-18. 